\title{
A ESTRUTURA ANALÍTICA DE PROJETO DINÂMICA (EAPD) COMO FERRAMENTA PARA MELHORAR O PLANEJAMENTO E EXECUÇÃO DOS PROJETOS
}

\author{
Luciano da Silva Bastos Sales (lucianofrc@gmail.com) - PPMEC, Universidade de Brasília \\ Sanderson César Macêdo Barbalho (scmbbr@yahoo.com.br) - PPMEC, Universidade de Brasília \\ Rodrigo Augusto (rodrigoaugusto.silvadossantos@mavs.uta.edu) - University of Texas at Arlington
}

\section{RESUMO}

A Estrutura Analítica de Projeto (EAP) é uma das principais ferramentas de gerenciamento de projetos, base para as atividades de execução. Ela parte da premissa que a divisão das entregas em componentes menores facilita o gerenciamento das mesmas, através de uma abordagem, descrita na literatura, como linear ou estática. Segundo a Dinâmica de Sistemas (DS), a fragmentação linear das entregas proposta pela EAP, traz como consequência a desconsideração dos aspectos intra-projetos, o que pode levar a perda de informação durante o planejamento, acarretando em possíveis problemas na execução. Esses problemas poderiam ser evitados, em muitos casos, com o uso do pensamento sistêmico. $O$ objetivo principal deste artigo é apresentar a incorporação na EAP dos princípios e ferramentas da DS para melhorar o desempenho da execução dos projetos, propondo a EAP Dinâmica (EAPD). Para isso, no referencial teórico são discutidos os problemas nos atuais modelos de gestão de projetos e apontadas algumas consequências dessas deficiências. Sob uma abordagem descritiva-exploratória foi realizada pesquisa mista por meio de um estudo de caso em um projeto público, com foco na implantação de uma infraestrutura de telecomunicações na Amazônia, e do desenvolvimento de uma pesquisa de levantamento entre profissionais de gerenciamento de projetos. Foram observados na prática que há uma amplo entendimento por parte dos gerentes de projeto que a EAP é importante para uma boa execução dos projetos e que muitos dos problemas de execução têm origem na forma como a EAP é desenvolvida e utilizada. Além disso, no âmbito do estudo de caso, foi utilizada a EAPD como ferramenta principal de planejamento do Projeto Infovias, parte do Programa Amazônia Conectada, o que representou uma melhoria consistente na execução do mesmo, se comparado com os seus estágios anteriores. Assim, muitos dos erros de execução foram antecipados, o que sugere que a utilização da dinâmica de sistemas pode complementar a modelagem tradicional, alavancando o planejamento e a execução dos projetos.

Palavras chave: Dinâmica de Sistemas; Gerenciamento de Projetos; Estrutura Analítica de Projeto; Arquétipos de Sistema.

Área: Gestão do Processo de Desenvolvimento de Produtos

\section{INTRODUÇÃO}

Segundo o PMI (2013), a EAP é a decomposição hierárquica do escopo do trabalho a ser executado pelas equipes de projeto a fim de alcançar os objetivos do projeto e criar as entregas exigidas. Esse tipo de decomposição está amparada por uma premissa, que afirma que, por mais que os projetos sejam únicos, suas partes constituintes já foram desenvolvidas antes por outros projetos, carregando em si experiências que facilitam o desenvolvimento de estimativas razoáveis (RODRIGUES; BOWERS, 1996). Porém, para esses mesmos pesquisadores, as estimativas desenvolvidas considerando as partes isoladas dos projetos, 
acabam por ignorar as forças intra-projetos, que podem levar a uma situação na qual o todo é maior que a soma de suas partes.

Senge (1996) afirma que, desde cedo aprendemos a dividir e fragmentar o mundo para tornar tarefas e assuntos complexos mais administráveis. Porém, ao fazer isso, pagamos um preço alto: "Não conseguimos mais perceber as consequências das nossas ações; perdemos a noção intrínseca de conexão com o todo". Segundo Williams (2000), por mais que as ferramentas de gerenciamento de projetos se baseiem na decomposição de suas partes fundamentais, durante a execução dos projetos ocorrem efeitos sistêmicos que devem ser levados em consideração no planejamento.

A DS é uma ferramenta que suporta o pensamento sistêmico levando em conta os relacionamentos entre as partes constituintes de um sistema no decorrer do tempo, sendo bastante útil para analisar a tomada de decisão, buscando uma compreensão mais ampla dos aspectos intra-projetos (HOFFENSON; SODERBERG, 2015). Embora os modelos da DS tenham sido amplamente aplicados na pesquisa acadêmica para resolver diferentes tipos de problemas de gerenciamento de projetos, a viabilidade de modelos da DS ainda requer uma investigação mais aprofundada caso-a-caso (OZCAN-DENIZ; ZHU, 2016).

Nesse contexto de problematização, a DS será analisada como ferramenta complementar ao desenvolvimento da EAP, visando a mitigação dos problemas na execução do gerenciamento de projetos (GP), através da seguinte questão de pesquisa: Como as ferramentas da DS podem ser integradas a EAP para melhorar o planejamento e a execução dos projetos? Assim, o objetivo deste artigo é o de identificar e descrever como a DS pode melhorar o desenvolvimento da EAP, alavancando a execução dos projetos, através da aplicação da modelagem soft em um estudo de caso.

Para alcançar esse objetivo, serão apresentados, na seção 2, os conceitos sobre a EAP, os problemas nas atuais abordagens de GP, a DS e a DS integrada ao GP; na seção 3, as questões metodológicas; na seção 4, a análise dos resultados; e, finalmente, na seção 5, as conclusões do trabalho proposto.

\section{REFERENCIAL TEÓRICO}

\subsection{A estrutura analítica de projeto}

Kenley e Harfield (2014) afirmaram que a EAP é um dos mais importantes conceitos de GP, possuindo relação direta com os aspectos práticos do planejamento e controle das atividades necessárias para completar um projeto. Para Kolltveit, Karlsen e Gronhaug (2007), a importância atribuída no GP, as entregas, aos custos e prazos, torna a EAP uma ferramenta central para o planejamento e controle dos projetos. Para Bachy e Hameri (1997), a EAP é a espinha dorsal do bom planejamento, execução e controle de um projeto.

Segundo Rodrigues e Bowers (1996), a construção de uma EAP precisa respeitar dois princípios básicos: completude, ou seja, todo o trabalho a ser desenvolvido pelo projeto, sem nenhuma exceção, precisa ser mapeado para algum pacote de trabalho na EAP; e hierarquia, pois a soma de todo o trabalho do projeto deve ser igual a soma dos elementos de todos os pacotes de trabalho decompostos.

Assim, uma EAP bem construída pode revelar-se crucial em muitos processos de GP, uma vez que divide o trabalho a ser desenvolvido em etapas, resultados e pacotes de trabalho, impactando diretamente na definição das atividades, no cronograma do projeto, na análise de riscos e respostas, e nas ferramentas de controle utilizadas nos projetos (SIAMIIRDEMOOSA; DINDARLOO; SHARIFZADEH, 2015). 


\subsection{Problemas nas atuais abordagens de gerenciamento de projetos}

Para contornar a sua complexidade natural, normalmente, os projetos são realizados por fases, ou seja, um produto é desenvolvido através de diversas atividades mais ou menos independentes que podem ser executadas e mantidas separadamente (PESONEN et al, 2008). Por exemplo, os modelos tradicionais de GP, tais como o Método do Caminho Crítico e a técnica PERT, descrevem o progresso dos projetos através de uma rede de atividades com durações estimadas para essas atividades. O problema é que esses modelos ignoram as iterações entre atividades e não as incorporam nas suas estimativas de duração, o que limita a capacidade do uso dessas técnicas de programação em processos de modelagem (LIN et al, 2008).

Um exemplo prático pode ser imaginado, quando a definição do desenho do produto é liberada com erros para a execução: quando o erro for percebido será necessário retornar para a atividade de desenho, ou seja, ocorreu uma iteração com impacto no cronograma e no orçamento, porém, esse tipo de iteração, normalmente, não é mapeada pelo Método do Caminho Crítico.

Segundo Chritamara, Ogunlana e Bach (2002), as técnicas tradicionais de planejamento de projetos não permitem antecipar os resultados das escolhas. Assim, os gerentes de projeto precisam tomar uma série de decisões baseadas em um planejamento linear, enquanto o mundo real é dinâmico por natureza. Esses pesquisadores sugerem a utilização de ferramentas da dinâmica de sistemas para complementar as boas práticas existentes.

A despeito da necessidade de utilização de ferramentas da DS, não foram encontradas evidências do seu uso nos principais Guias de GP: o PMBOK 5 ou o PRINCE2.

\subsection{Dinâmica de sistemas}

Jay Forrester é reconhecido como o pioneiro no desenvolvimento das ideias que deram origem à Dinâmica de Sistemas (DS), difundidas com o lançamento do livro Industrial Dynamics, em 1961 (FERNADEZ, 2003). A DS possui um conjunto de ferramentas que facilita a compreensão das estruturas e da dinâmica de sistemas complexos, além de ser um método de modelagem que permite o desenvolvimento de simulações que antecipam os resultados das ações a serem tomadas nesses sistemas (SALES et al, 2016).

Assim, quando duas ou mais variáveis formam um loop (ciclo completo) fechado de relações, onde a primeira influencia a segunda, a segunda uma terceira, e assim por diante, até que a última variável gere uma influência sobre a primeira variável considerada, em um ciclo sem início ou fim, temos um loop de feedback - ciclo de retroalimentação (FORRESTER, 2009).

Esses ciclos de retroalimentação, conforme apresentado na Figura 1, são responsáveis pelos mecanismos de reforço (positivo) ou de equilíbrio (negativo) (SENGE, 1996). Os ciclos de reforço possuem um comportamento mais previsível, pois as variáveis reforçam ou aceleram a mudança inicial, além de possuírem comportamento exponencial, seja crescente ou decrescente, que poderá ocorrer de forma indefinida, a não ser que sejam introduzidas variáveis de restrição. Já os loops de equilíbrio ocorrem quando existe uma relação ou quando um número ímpar de relações do loop for negativo, restringindo a direção inicial da mudança das variáveis. 


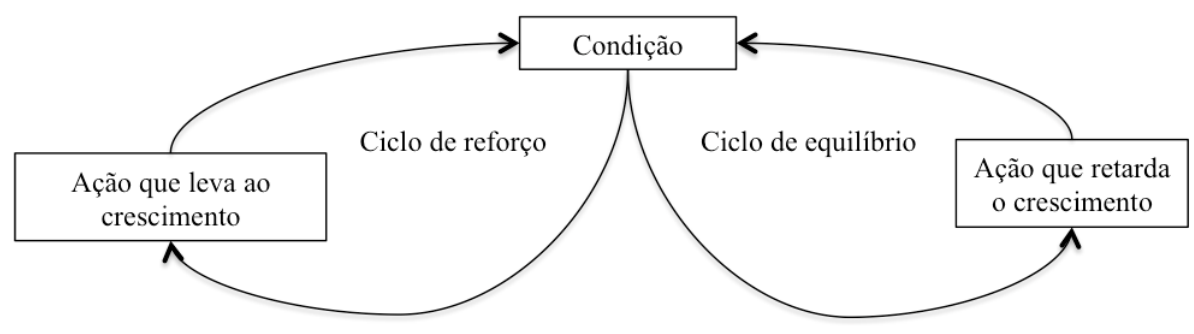

As técnicas de modelagem da DS podem ser classificadas em dois tipos: a soft, mais subjetiva e focada no aspecto qualitativo e no aprendizado sistêmico; e a hard, com um foco mais quantitativo e voltada à simulação e tomada de decisão (FERNANDEZ, 2003).

\subsection{Dinâmica de sistemas integrada ao gerenciamento de projetos}

Os modelos da DS são normalmente implantados para resolver problemas complexos (OZCAN-DENIZ; ZHU, 2016). Na área de projetos, os comportamentos mais complexos geralmente surgem das interações entre os componentes do sistema, e não a partir da complexidade dos próprios componentes (PESONEN et al, 2008).

O uso da DS no GP tem por objetivo compreender e considerar todos os processos de feedbacks responsáveis pelo comportamento do sistema, tanto do ponto de vista da integração das entidades internas, quanto do ponto de vista do contexto ambiental externo ao qual essas entidades estão submetidas (RODRIGUES; BOWERS, 1996).

Tipicamente, a estrutura do modelo de DS a ser utilizado em projetos deve incluir os seguintes aspectos: (1) as características principais desse projeto (pacotes de trabalho ou atividades); (2) seu ciclo de retrabalho (como os pacotes de trabalho interagem no tempo); (3) os feedbacks de controle do projeto (os pontos onde os projetos serão mensurados); e (4) os prováveis efeitos colaterais (ou seja, o resultados das ações executadas para superar o gap entre o desempenho do projeto e seu alvo) (OZCAN-DENIZ; ZHU, 2016).

Senge (1996), afirma que dentro da perspectiva do pensamento sistêmico determinados padrões e estruturas ocorrem de forma recorrente. O referido pesquisador chama esses padrões de "arquétipos de sistema", mostrando que nem todos os problemas gerenciais são específicos.

Pelo contrário, as ferramentas da dinâmica de sistemas são a base para a identificação dos arquétipos, cujo entendimento contribui para a solução dos problemas de fracionamento do conhecimento, típicas das decomposições utilizadas no GP. Cada arquétipo possui uma estrutura típica, sendo possível, a partir desses padrões identificados, tomar melhores decisões e prevenir erros a partir da eliminação dos fatores limitadores.

\section{METODOLOGIA}

$\mathrm{Na}$ elaboração deste estudo, sob uma abordagem descritiva-exploratória, foi realizada pesquisa mista por meio de um estudo de caso em um projeto público e do desenvolvimento de uma pesquisa de levantamento entre profissionais de gerenciamento de projetos.

A pesquisa de levantamento foi realizada para verificar se há entendimento por parte dos gerentes de projeto que a EAP é importante para uma boa execução dos projetos e se esses 
gerentes concordam que os problemas de execução em seus projetos tiveram origem na EAP desenvolvida.

Foi utilizada a ferramenta de survey do google, com o convite para a participação na pesquisa enviado para grupos de e-mail sobre gerenciamento de projetos, com 6 perguntas: (1) Você possui quanto tempo de experiência em projetos? (2) Quais os principais problemas encontrados na execução dos projetos que você vem participando? (3) Você utiliza a EAP como ferramenta de planejamento dos seus projetos? (4) Você concorda que a EAP (ferramenta de planejamento) é importante para uma boa execução dos projetos? (5) Você entende que os problemas de execução já apontados por você tiveram origem na criação/uso da EAP? (6) Quais os problemas de execução apontados por você tiveram origem na criação/uso da EAP?

Para o estudo de caso, a unidade de análise é o processo de planejamento e execução do Projeto Infovias (PI) do Programa Amazônia Conectada (PAC). O PAC é uma iniciativa do Estado Brasileiro, com foco na implantação de uma infraestrutura de telecomunicações na Amazônia, através de 05 (cinco) infovias utilizando cabos de fibra óptica subfluvial, que irão interligar 52 municípios no Estado do Amazonas, através de 05 (cinco) projetos.

A análise dos dados ocorreu através da utilização da proposição teórica de Ozcan-Denis e Zhu (2016) para uso da DS em projetos e da construção da explicação, comparando-se as conclusões do estudo de caso com a proposição teórica. É apresentado, na Tabela 1, um resumo dos aspectos metodológicos utilizados nesta pesquisa.

Tabela 1. Resumo dos aspectos metodológicos desta pesquisa. Fonte: Os autores.

\begin{tabular}{|c|c|}
\hline Caracterização da Pesquisa & Organização da Pesquisa \\
\hline Abordagem metodológica: Mista & Objeto empírico: PI do PAC \\
\hline Tipo de Pesquisa: Descritiva-exploratória & Quantidade de observações: Estudo de caso único \\
\hline $\begin{array}{l}\text { Técnica de Investigação: Estudo de Caso e pesquisa } \\
\text { de levantamento }\end{array}$ & $\begin{array}{c}\text { Unidade de análise: processo de planejamento e } \\
\text { execução do PI }\end{array}$ \\
\hline $\begin{array}{l}\text { Fontes de Coleta de Dados: Survey, Análise } \\
\text { documental e Observação participante }\end{array}$ & $\begin{array}{l}\text { Unidade de observação: Estrutura Analítica do PI e } \\
\text { demais documentos de planejamento desse projeto }\end{array}$ \\
\hline $\begin{array}{l}\text { Análise dos dados: Através das proposições teóricas } \\
\text { propostas por Ozcan-Deniz e Zhu e da construção da } \\
\text { explicação }\end{array}$ & $\begin{array}{c}\text { Enfoque de Observação: Explorar a EAP para } \\
\text { confirmar ou refutar as descobertas do referencial } \\
\text { teórico }\end{array}$ \\
\hline
\end{tabular}

\section{ANÁLISE DOS RESULTADOS}

Os principais resultados da pesquisa de levantamento podem ser vistos na Figura 2. O survey ficou disponível por 15 dias na internet e foi respondido por 44 profissionais de projetos. É possível observar na Figura 2: (a) o tempo de experiência dos gerentes respondentes; (b) que 93\% dos respondentes utilizam a EAP como ferramenta de planejamento; (c) que 100\% dos respondentes concordam que a EAP é importante para uma boa execução dos projetos; (d) que $65,1 \%$ dos respondentes entende que os problemas de execução em seus projetos têm origem na criação/uso da EAP.

Assim, a partir dos resultados do survey é possível verificar um viés de compreensão por parte dos gerentes de projeto da importância da EAP para a execução bem-sucedida de um projeto, bem como, o entendimento que muitos dos problemas ocorridos durante a execução, têm origem na EAP. Há nesse resultado, uma tendência que confirma o que foi apresentado no 
referencial teórico, parecendo que há espaço para melhoria na EAP, o que foi feito no âmbito desta pesquisa através da modelagem soft da DS.

Figura 2. Resultado da pesquisa de levantamento em 4 partes. Fonte: Os autores.

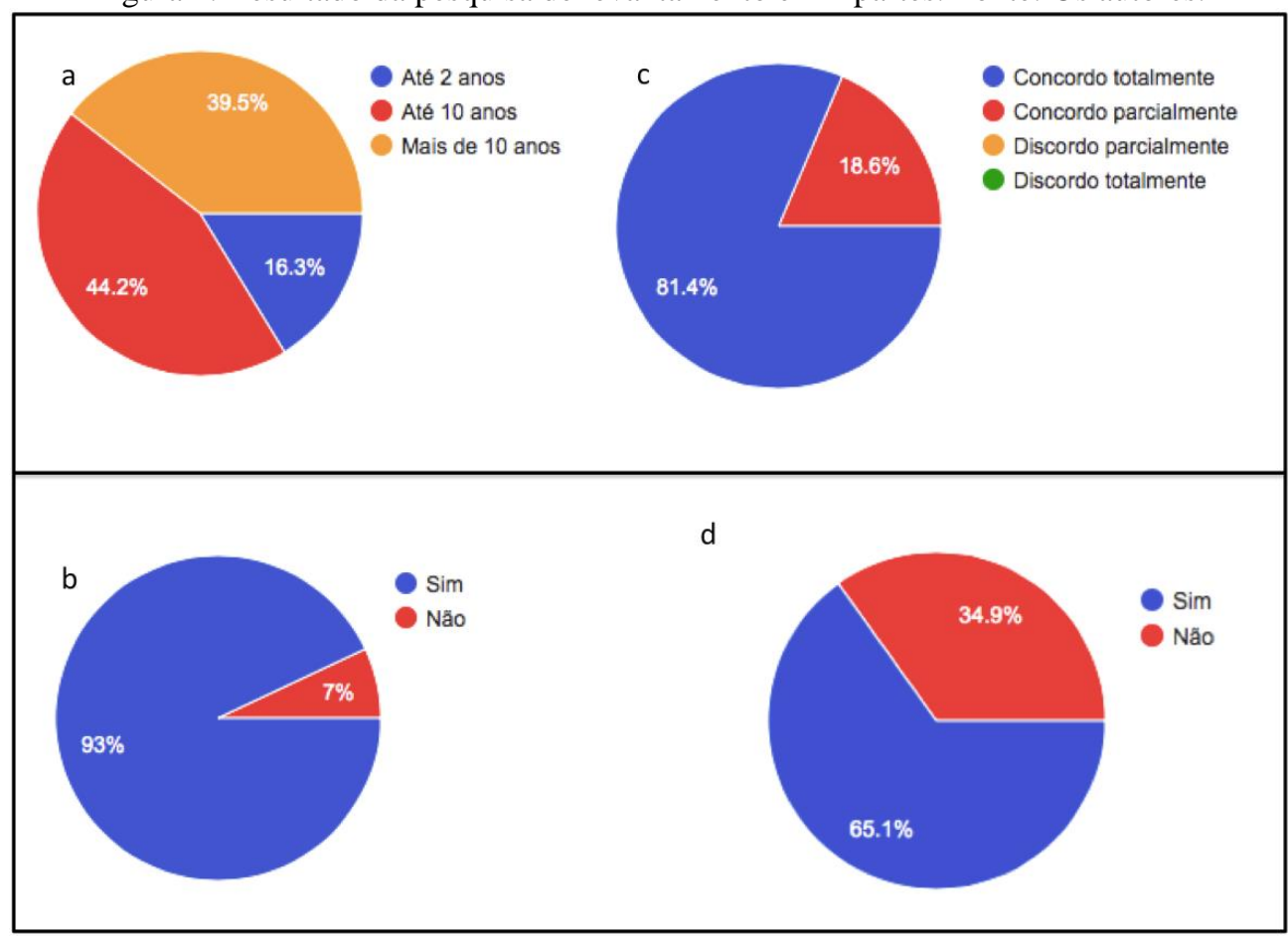

Com relação as perguntas 2 e 6 do survey, a maior parte das respostas, apontou que erros na execução dos projetos, relacionados ao cronograma, orçamento e limitações do escopo, tiveram origem na criação/uso da EAP.

A partir de agora serão expostos os resultados obtidos através do uso da DS, que se mostrou eficiente em antecipar eventuais problemas e diagnosticar os arquétipos apontados por Senge (1996) no diagrama de enlace-causal desenvolvido. O primeiro passo foi a utilização da EAP do Projeto Infovias, a mais completa dos documentos analisados, através da identificação de suas entregas principais: 1) implantação dos cabos ópticos subfluviais; 2) implantação dos equipamentos (Figura 3).

Para a construção do diagrama de enlace causal, partimos do pacote de trabalho que marca o início do trabalho em cada infovia: A aquisição dos cabos ópticos. Durante o procedimento, para cada pacote de trabalho, foi identificada a sua interação com os outros pacotes de trabalho e se havia algum feedback de controle e/ou efeitos colaterais, que precisavam ser considerados para fins de construção das relações de causalidade. 
Figura 3. Estrutura Analítica do Projeto Infovias. Fonte: Os autores.

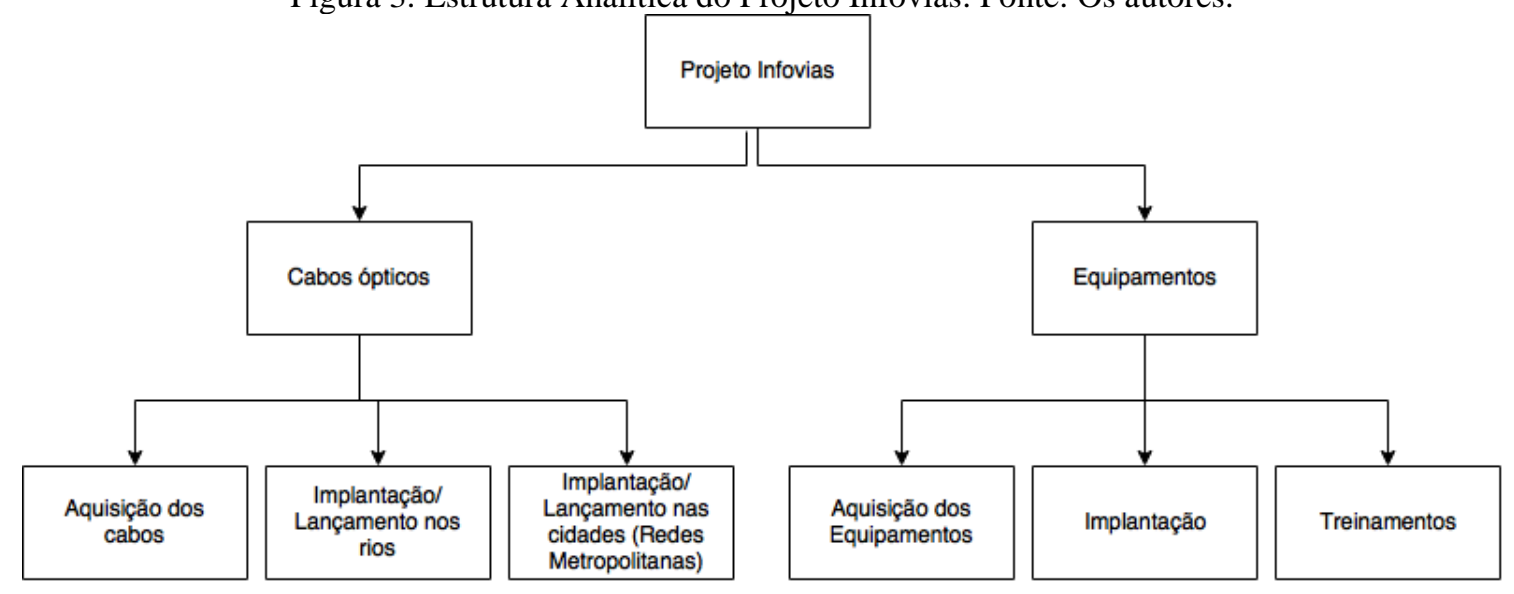

Como exemplo, seguindo a lógica mostrada na Figura 4, partindo do pacote de trabalho "aquisição dos cabos", há uma interação lógica com o procedimento de lançamento (quanto maior a aquisição de cabos, maior a eficácia). O lançamento precisa ser eficaz - feedback de controle (a meta está sendo mensurada pelo número de cidades atendidas). Assim, quanto maior a eficácia do lançamento, maior o número de cidades atendidas. Quanto mais cidades atendidas, maior o impacto social (benefício do programa).

Os resultados do impacto social é a métrica para conseguir recursos financeiros e, assim adquirir mais cabos, gera-se um ciclo de reforço. Porém, ao se lançar um cabo óptico subfluvial que interliga cidades, faz-se necessário criar redes metropolitanas (MAN) para atender à população e aos órgãos envolvidos. Ou seja, quanto mais cidades atendidas maior o esforço em se construir MAN. O efeito colateral ocorre sobre as cidades atendidas: o processo de construção de redes nas cidades, a despeito de se ter uma complexidade menor que o lançamento do cabo nos rios, consome um esforço maior, consumindo o tempo das equipes e diminuindo o número das cidades atendidas: eis um ciclo de equilíbrio.

Foram identificados dois arquétipos na EAPD desenvolvida: o "Limites ao Crescimento" e a "Transferência de responsabilidade para o interventor".

Senge (1996) afirma que no primeiro arquétipo um processo se autoalimenta para produzir um período de expansão. Porém, uma condição desse processo de reforço gera uma ação de redução do crescimento (ciclo de equilíbrio 1, da Figura 4), que deve desacelerar o ritmo inicial, parar os resultados e até de certo modo reverter, iniciando um colapso. A forma de proteção é a seguinte: não adianta forçar o processo de reforço, deve ser eliminada (ou enfraquecida) a fonte de limitação. A ação tomada no Programa para evitar o problema que já havia ocorrido nos outros dois estágios de execução, foi a separação dessa entrega (MAN), que deu origem a um novo projeto: a eliminação da fonte de limitação (primeira mudança no escopo do projeto).

O segundo arquétipo ocorre quando interventores externos são chamados para resolver problemas, e faz isso com tanto sucesso que as pessoas do sistema não aprendem a lidar com esses problemas por si próprias (SENGE, 1996). Esse arquétipo possui a seguinte estrutura: dois ciclos de equilíbrio (ciclo de equilíbrio 2 e 3, da Figura 4) que gera um ciclo de reforço (ciclo de reforço 3, da Figura 4) que vicia o sistema, criando uma série de problemas. A solução para enfrentar esse arquétipo: ampliar as capacidades dos agentes internos para que estes resolvam os seus problemas. No caso do Projeto, houve a segunda mudança no escopo, pois as capacitações foram adiantadas, já que há um delay (atraso) para que os treinamentos formem pessoas com experiência. Ou seja, foi evitado o uso de equipe externa durante a operação, realizando as capacitações locais antes do sistema entrar em operação. 
A identificação desses dois arquétipos resultou não só numa mudança do escopo do projeto, mas, também, numa mudança na programação do cronograma, como consequência das mudanças nos pacotes de trabalho da EAP. Isso só se tornou possível graças à identificação das interações entre os pacotes de trabalhos e os efeitos colaterais dessas interações. Como esses erros não são percebidos na EAP, usada como base para uma série de decisões do projeto como a construção do cronograma e a orçamentação, o problema vai se agravando conforme a execução vai avançando.

Figura 4. EAPD do Projeto Infovias. Fonte: Os autores.

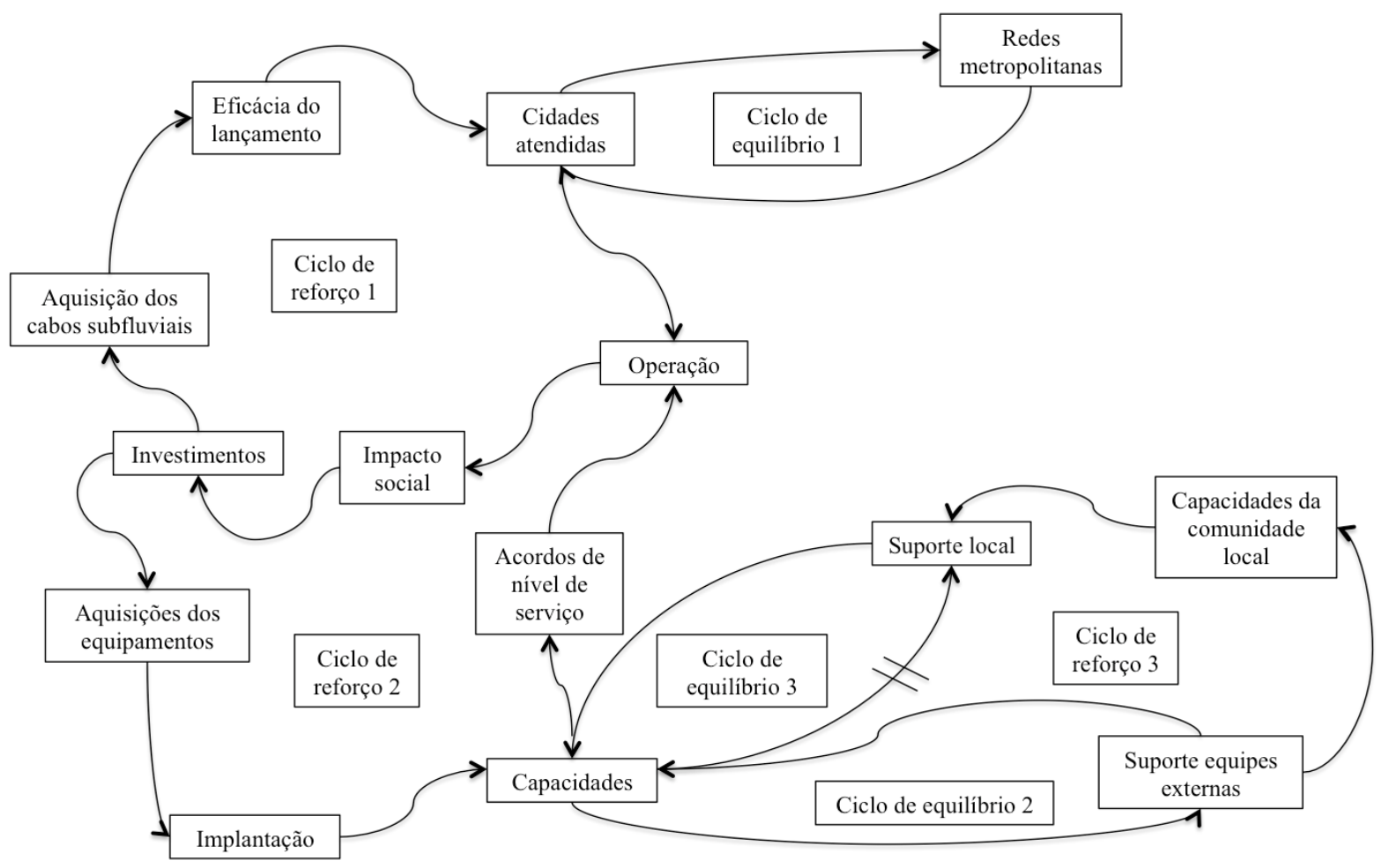

\section{CONCLUSÃO}

O objetivo deste artigo foi o de identificar e descrever como a DS pode melhorar o desenvolvimento da EAP, alavancando a execução dos projetos, através da aplicação da modelagem soft em um estudo de caso. A literatura apontou alguns problemas com as atuais modelagens de gerenciamento de projetos, devido a sua estrutura linear e estática. Esses problemas podem levar a decisões equivocadas que geram problemas na execução dos projetos, o que poderá ocasionar, por exemplo, retrabalho e custos maiores.

A recomendação proposta através dos resultados deste estudo é a de que se deve incorporar a DS ao modelo tradicional de EAP (EAPD), através da modelagem soft, que permite a identificação de ciclos de feedback e de arquétipos já mapeados na literatura. Os ciclos de feedback e os arquétipos, se não identificados durante o planejamento, levam a problemas na execução dos projetos. Assim, foi percebido no estudo de caso, que ao desconsiderar as interações sistêmicas entre os pacotes de trabalho, os times de projeto não conseguem identificar ciclos de feedback e outras limitações externas que podem levar a erros nas decisões dos projetos, gerando cronogramas equivocados e custos ocultos, justamente os 
principais problemas de execução apontados pelos gerentes de projeto com origem na criação/uso da EAP, segundo o survey.

Em suma, conclui-se, baseado no estudo de caso, que a EAPD pode melhorar a tomada de decisão nos projetos uma vez que: a) consegue antecipar o impacto das decisões tomadas durante o planejamento (evidenciado através da identificação dos dois arquétipos na modelagem soft do projeto Infovias); b) permite que a equipe do projeto identifique iterações e influências entre pacotes de trabalho (evidenciado, também, através do trabalho desenvolvido pela equipe na construção do diagrama de enlace-causal); c) auxilia no desenvolvimento de cronogramas mais realistas (evidenciado através da necessidade de alteração do escopo do projeto Infovias, o que levou a uma mudança no cronograma do projeto); e d) evita o retrabalho (uma vez que os erros foram percebidos antes mesmo de ocorrerem).

Durante o desenvolvimento deste artigo verificamos a existência de algumas limitações, sendo a principal delas referente ao fato da pesquisa estar restrita a um único caso, o que não permite a generalização de qualquer resultado. Ademais, este artigo suscita uma série de trabalhos futuros, dentre os quais vale mencionar a aplicação da EAPD em outros projetos para generalizar os resultados, a modelagem dos arquétipos que podem influenciar os projetos, e a identificação de outras ferramentas de projetos que possam ser integradas com a DS para otimizar a execução dos mesmos.

\section{REFERÊNCIAS}

BACHY, G.; HAMERI, A. What to be implemented at the early stage of a large-scale project: International Journal of Project Management, v. 14, n. 4, p. 211-218, 1997.

CHRITAMARA, S., OGUNIANA, S. System dynamics modeling of design and build construction projects: Constrution Innovation, n. 2, p. 269-295, 2002.

FERNANDEZ, A. "Scorecard Dinâmico: Em direção à integração da Dinâmica de Sistemas com o Balanced Scorecard. 2003. Tese de Doutorado. COPPE/UFRJ, Rio de Janeiro, Brasil.

FORRESTER, J. Some basic concepts in system dynamics. In: Report Nr D-4894. Massachusetts Institute of Technology, Sloan Schoolof Management, 2009.

HOFFENSON, S.; SODERBERG, R. Systems thinking in tolerance and quality-related design decision-making: Procedia CIRP, n. 27, p. 59-64, 2015. Goteborg, Sweden.

KENLEY, R.; HARFIELD, T. Reviewing the IJPM for WBS: the search for planning and control: Procedia - Social and Behavioral Sciences, n. 119, p.887-893, 2014.

KOLLTVEIT, B.; KARLSEN, J.; GRONHAUG, K. Perspectives on Project management: International Journal of Project Management, n. 25, p. 3-9, 2007.

LIN, J., CHAI, K., WONG, Y., BROMBACHER, A. A dynamics model for managing overlapped iterative product development: European Journal of Operational Research, n. 185, p. 378-392, 2008.

OZCAN-DENIZ, G., ZHU, Y. A system dynamics model for construction method selection with sustainability considerations: Jornal of Cleaner Production, n. 121, p. 33-44, 2016.

PESONEN, L.; SALMINEN, S.; YLÉN, J.; RIIHIMAKI, P. Dynamic simulation of products process: Simulation Modelling Practice and Theory, n. 16, p. 1091-1102, 2008.

PROJECT MANAGEMENT INSTITUTE (PMI). PMBOK. USA: Author, 2013. 
RODRIGUES, A.; BOWERS, J. The role of system dynamics in project management: International Journal of Project Management, n.14, p. 213-220, 1996.

SALES, L., ROSES, K., PRADO, H. Application of dynamic balanced scorecard in the Brazilian Army information technology governance: Gestão e Produção, n. 23, pp 757-770, 2016.

SENGE, P. M. A Quinta Disciplina: A arte e a prática da Organização que aprende”. Rio de Janeiro, RJ: Best Seller, 1996.

SIAMI-IRDEMOOSA, E.; DINDARLOO, S.; SHARIFZADEH, M. Work breakdown structure (WBS) development for underground construction: Automation in Construction, n. 58, p. 85-94, 2015.

WILLIAMS, T. Safety regulation changes during projects: the use of system dynamics to quantify the effects of change: International Journal of Project Management, n. 18, p. 23-31, 2000. 